Radial and Nonradial Pulsations as Probes of Stellar Physics

ASP Conference Series, Vol. 259, 2002

C. Aerts, T.R. Bedding, 63 J. Christensen-Dalsgaard, eds.

\title{
A Photometric Study of the $\delta$ Scuti Star UV Trianguli
}

\author{
Z. L. Liu, A. Y. Zhou \\ National Astronomical Observatories, CAS, Beijing 100012, China \\ E. Rodríguez \\ Instituto de Astrofisica de Andalucia, CSIC, P. O. Box 3004, E-18080 \\ Granada, Spain
}

\begin{abstract}
UV Tri was observed photometrically from 1999 to 2000 . A period analysis of the data reveals two pulsation frequencies at 9.3299 and $10.8483 \mathrm{~cd}^{-1}$. From $u v b y H_{\beta}$ data we derive $V=11.085, b-y=$ $0.215, m_{1}=0.169, c_{1}=0.783$, and $\beta=2.775 \mathrm{mag}$. With these color indices and some calibrations, we obtain $M_{v}=2.44 \mathrm{mag}, M_{\text {bol }}=2.27$ $\mathrm{mag}, T_{\mathrm{eff}}=7500 \mathrm{~K}, \log L / L_{\odot}=0.99$ and a distance of $\sim 536 \mathrm{pc}$.
\end{abstract}

\section{Introduction}

UV Trianguli ( $V=11^{\mathrm{m}} 0, \mathrm{P}=\sim 0{ }^{\mathrm{d}} 1$ ) was found to be a $\delta$ Scuti star by Shaw et al. (1983) when they observed a nearby eclipsing binary V Tri. Due to a lack of data these authors could not give more results, but they pointed out the possibility of multiple periods. In order to obtain accurate frequency information and some physical parameters, new observations were made.

\section{Photometric $V$ observations and period analysis}

From November 17, 1999 to January 14, 2000 UV Tri was observed, using the CCD photometer (Wei et al., 1990) attached to the $85-\mathrm{cm}$ telescope at the Xinglong Station of the Beijing Astronomical Observatory. During the observations, GSC 2293-1028 was used as the comparison star. GSC 2293-1422, 2293-1027, 2293-1461 and 2293-1456 were used as check stars. From October 24 to November 20, 2000 the star was re-observed using the WET high-speed 3-channel photometer (Jiang \& Hu, 1998) on the same telescope. GSC 2293-1331 was used as comparison. The Johnson $V$ filter was used throughout the observations. We resampled the data into bins of $60 \mathrm{~s}$. Finally, we obtained 6326 measurements (a length of $4.7772 \mathrm{~d}$ ) in 18 nights covering a period of $370 \mathrm{~d}$. The measuring standard error of data obtained using the CCD photometer is about $0 . \mathrm{m} 01$. No evidence of variability of the comparisons was found within the error ranges. We performed a period analysis to the whole $V$ data and detected two pulsation frequencies at 9.3299 and $10.8483 \mathrm{~cd}^{-1}$ with amplitudes of $0 .{ }^{\mathrm{m}} 026$ and $00^{\mathrm{m}} 010$, respectively. The two frequencies fit the observations very well. 


\section{3. $u v b y H_{\beta}$ observations and determination of the physical parameters}

(1) In order to derive the physical parameters of UV Tri, the Strömgren $u v b y H_{\beta}$ data were collected using the $90-\mathrm{cm}$ telescope at the Sierra Nevada Observatory (Spain) in 2000. HD 9483, HD 9445 and HD 8826 were used as comparison stars. For UV Tri, 55 uvby and $4 H_{\beta}$ data points were obtained. For the three comparisons, $\left(u v b y, H_{\beta}\right)$ sets of $(91,7),(20,4)$ and $(21,4)$ points were measured. Our data have been transformed into the standard system. The results are listed in Table 1 . The typical deviations obtained in the transformation equations were $0.011,0.007,0.009,0.009$ and $0.008 \mathrm{mag}$ for $V, b-y, m_{1}, c_{1}$ and $\beta$, respectively. To evaluate our results, the corresponding values of Hauck \& Mermilliod (1998) are adopted. Our results are in good agreement with theirs.

Table 1. Magnitude and colour indices of UV Tri and 3 comparisons.

\begin{tabular}{clllll}
\hline Object & $V / \sigma$ & $b-y / \sigma$ & $m_{1} / \sigma$ & $c_{1} / \sigma$ & $\beta / \sigma$ \\
\hline UV Tri & $11.085(21)$ & $0.215(15)$ & $0.169(14)$ & $0.783(41)$ & $2.775(16)$ \\
HD 9483 & $8.091(4)$ & $0.116(4)$ & $0.161(5)$ & $0.985(15)$ & $2.835(9)$ \\
HD 9445 & $8.482(4)$ & $0.252(5)$ & $0.162(5)$ & $0.779(15)$ & $2.722(7)$ \\
HD 8826 & $7.998(5)$ & $0.274(4)$ & $0.148(4)$ & $0.459(11)$ & $2.669(12)$ \\
HD 9483 & 8.06 & 0.120 & 0.141 & 0.979 & 2.838 \\
HD 9445 & 8.50 & 0.255 & 0.167 & 0.788 & 2.717 \\
HD 8826 & 8.01 & 0.267 & 0.163 & 0.456 & 2.671 \\
\hline
\end{tabular}

(2) Depending on color indices and applying the calibrations of Crawford (1979) and Moon \& Dworetsky (1985), we obtained $M_{v}=2^{\mathrm{m}} 44 \pm 0.10$ and $T_{\text {eff }}=$ $7500 \pm 100 \mathrm{~K}$. Other calibrations consistently confirmed the values.

(3) Shaw et al. (1983) estimated the spectral type of UV Tri to be A 3. If so, the bolometric correction is -0.17 (Schmidt-Kaler, 1982). If zero interstellar reddening was assumed, the absolute bolometric magnitude of UV Tri is: $M_{\text {bol }}$ $=2^{\mathrm{m}} 27 \pm 0.10$. Then we obtained $\log L / L_{\odot}=0.99 \pm 0.05$.

(4) Using the $V$ magnitude (11. 085$)$ and $M_{v}=2{ }^{\mathrm{m}} 44$, we obtained the distance of UV Tri, $R=535.8$ pc by $M_{v}=V+5-5 \log R$.

Acknowledgments. LZL thanks the NSFC (China) and ER acknowledges the Junta de Andalucia and the DGES under project PB96-0840.

\section{References}

Crawford, D.L. 1979, AJ, 84, 1858

Hauck, B. \& Mermilliod, M. 1998, A\&AS, 129, 431

Jiang, X.J. \& Hu, J.Y. 1998, Acta Astron. Sinica, 39, 438

Moon, T.T. \& Dworetsky, M.M. 1985, MNRAS, 217, 305

Schmidt-Kaler, Th. 1982, Landolt-Börnstein New Series, VI/2b

Shaw, J.S., et al. 1983, IBVS, 2289

Wei, M.Z., Chen, J.S., \& Jiang, Z.J. 1990, PASP, 102, 698 